FERNANDO RIOS

ISA MARIA F. ROSA GUARÁ*

\title{
A quantas juventudes temos que atender?
}

Jovem ou jovens. Juventude ou juventudes.

O que existe de comum entre George Bush e Ozama Bin Laden? Evidentemente, ambos foram jovens. E que mais?

Eles têm uma incrível determinação: utilizam tudo o que está ao seu alcance, dos gestos de violência (verdadeiros) aos gestos de amizade (verdadeiros e falsos) para atingir seus objetivos, que são bem diferentes.

Quais as diferenças entre um jovem fundamentalista islâmico, um evangélico, um budista, um católico. São Muitas. Cada um deles tem sua visão de mundo particular e se comporta em função disso.

Mesmo considerando que a sociedade capitalista oferece aos jovens brasileiros modelos semelhantes de consumo e... felicidade, através dele, o capitalismo, precisamos considerar os muitos jovens e as muitas juventudes presentes neste momento brasileiro.

Temos diferentes jovens em São Paulo, no Rio e Janeiro, em Minas Gerais, Pernambuco, no Amazonas, no Rio Grande do Sul.

Temos cidades e áreas rurais. Temos jovens instruídos e sem instrução. Temos classes sociais $A, B, C, D$ e E. e, para cada uma delas, suas características, seus modelos, suas regras, seus desejos. Temos jovens filhos do poder econômico e político $e$ jovens filhos de assalariados máxima ou minimamente, embora os minimamente sejam em maior número.

Mas... de que jovem nós estamos falando? Do sexo masculino ou feminino? Negro, branco, moreno, louro, índio, asiáticodescendente etc. etc. etc?

As possíveis combinações de jovens são aquelas combinações dos seres humanos.

Nenhum retrato corresponde à realidade. Ela é muito maior do que nossa vã pretensão de abrangê-la. As estatísticas nos mostram números. Mas nenhum jovem é um número. É um indivíduo e suas múltiplas circunstâncias.

Por isso, este mosaico. Verdadeiramente, um mosaico de propostas de conhecimento das múltiplas realidades que envolvem a juventude. E cada uma delas tem uma demanda específica, apesar de, estatisticamente, existir algo comum: uma mesma faixa etária e um imenso desejo de consumo.

Quando ouvimos Paulinho da Viola cantar...
Consumir é viver

Conviver é sumir

... e olhamos ao redor, não encontramos uma reposta adequada a uma simples pergunta: o que eu faço com o outro? Talvez, para muitas pessoas, haja duas perguntas anteriores: existe um outro de mim? Se existe, quem é ele?

0 que comumente acontece é que não percebemos o outro, ele quase não existe, ou melhor, costuma ser considerado apenas para servir. Servir a um sujeito hipertrofiado, individualista, consumidor, habitando cada indivíduo e, por extensão, cada jovem.

É nessa direção que, acreditamos, deve caminhar a reflexão. Por isso, além desta revista inteira, trazemos alguns filmes, sites e livros. Para que eles nos ajudem a, olhando o passado, compreender o presente e intuir e propor um futuro melhor.

Entre o James Dean de Juventude transviada e o Marlon Brando de $O$ Selvagem, modelos de rebeldia do século passado, $e$ Bicho de sete cabeças, Billy Elliot e Doze trabalhos, há muitos mundos de diferença. Muitos mundos que aconteceram no século XX e que nos deixam cheios de dúvidas e temores neste começo do internético século XXI.

E então, o que devemos fazer com e para as nossas juventudes? Elas podem conviver pacificamente?

Onde estão as ameaças e as possíveis soluções?

Certamente, educação é uma das soluções. Educação formal e informal. Pública ou privada.

Conhecimento é outra.

Porém, enquanto refletimos, no meio disso tudo, continuamos a ouvir os jovens pedindo um lugar ao sol e outro à sombra. Como todos nós, adultos temerosos, cada vez mais solitários, ilhados em circunstâncias, da qual a mais enfática se chama violência.

Estamos preparados para atendê-los ou eles nos obrigarão $a$ isso?

\footnotetext{
* Fernando Rıos é jornalista, publicitário, cientista social e consultor em Comunicação Organizacional Integrada. ISA MARIA F. RoSA GUARÁ é pedagoga, psicopedagoga, doutora e mestre em Serviço Social (PUC-SP), consultora de projetos sociais e de educação e coordenadora editorial dos Cadernos CENPEC.
} 


\section{Filmes}

\section{AMOR, SUBLIME AMOR.}

DIREÇÃO: JEROME ROBBINS E ROBERT WISE

MÚSICA: LEONARD BERNSTEIN E SAUL CHAPLIN

ATORES/DAnÇARINOS: Natalie Wood (Maria), Richard Beymer (Tony), Russ Tamblyn (Riff), Rita Moreno (Anita), George Chakiris (Bernardo), Simon Oakland (Tenente Schrank), Ned Glass (Médico), William Bramley (Oficial Krupke), Tucker SMITH (ICE)

TONY MORDENTE (ACTION).

MUSICAL, EUA, 1961, 155 MINUTOS, CORES.

Um dos mais belos musicais norte-americanos, West Side Story, no original, transporta Romeu e Julieta para Nova lorque. Montechios e Capuletos se transformam em duas gangues que disputam uma área: Sharks, de porto-riquenhos, e Jets, de brancos de origem anglo-saxônica. O líder dos Jets, se apaixona por Maria, irmã de Riff, líder dos Sharks.

\section{BILLY ELLIOT}

DIREÇÃO: STEPHEN DALDRY

atoreS: Julie Walters (Mrs. Wilkinson), Jamie Bell (Billy Elliot), Jamie Draven (Tony), Gary Lewis (Pai), Jean Heywood (Avó), Stuart Wells (Michael), Nicola BlackWell (DebBie)

DRAMA, INGLATERRA, 2000, 111 MINUTOS, CORES.

Billy Elliot, é a história de Jamie Bell, de 11 anos, que vive numa pequena cidade inglesa, onde o principal meio de sustento são as minas da cidade. É obrigado a treinar boxe por imposição do pai; mas sua grande paixão é o balé, que passa a dançar escondido de sua família, mantendo sempre o sonho de se tornar um grande bailarino. Recebeu três indicações ao Oscar.

\section{BICHO DE SETE CABEÇAS}

DIREÇÃO: LAÍS BODANSKY

atores: Rodrigo Santoro (Neto), Othon Bastos (Seu Wilson), Cássia Kiss (MÃe de Neto), Jairo Mattos, Caco Ciocler, Luís Miranda, Valéria Alencar, Altair lima, Linneu Dias, Gero Camilo, Marcos Cesana, Sílvia lourenço, Daniela Nefussi.

DRAMA, BRASIL, 2.000, 80 MINUTOS, CORES.

Pai e filho são adversários. Seu Wilson, um representante da classe média, não entende e nem pretende entender seu filho, Neto. A relação é extremamente conflituosa. Ela chega ao máximo quando Seu Neto resolve mandar Neto para um manicômio. Aos poucos, ambos se destroem.

\section{JUNO}

DIRETOR: JASON REITMAN

atores: Ellen Page (Juno MacGuff), Michael Cera (Paulie Bleeker), Jennifer Garner (Vanessa loring); Jason Bateman (Mark Loring); Allison Janney (Bren MacGuff); J.K. Simmons (Mac MacGuff); Olivia Thirlby (Leah); Eileen Pedde (Gerta Rauss); Rainn Wilson (Rollo); Daniel Clark (Steve Rendazo); Darla Vandenbossche (Mãe de Bleeker).

COMÉDIA, EUA, 2007, 96 MINUTOS, CORES.

Juno, uma adolescente de 16 anos, fica gráfica de seu amigo Paulie, com quem tranzou uma vez. Ela se acha incapaz de ter cuidar da c riança e resolve entregá-la a um casal que tenha melhores condições de criá-la. Um excelente tema para tantas garotas que engravidam e, certamente, terão suas vidas completamente alteradas. Sem falar dos inúmeros problemas que a criança terá que enfrentar. 


\section{JUVENTUDE TRANSVIADA}

DIREÇÃO: NICHOLAS RAY

atores: James dean (Jim Stark), Natalie Wood (Judy), Sal Mineo (John Crawford), Jim Backus (Frank Stark), Ann Doran (Sra. Stark), Corey Allen (Buzz Gunderson), William Hopper (Pai de Judy), Rochelle Hudson (MÃe de Judy), Dennis Hopper (GOON).

DRAMA, EUA, 1955, 111 MINUTOS, BRANCO E PRETO.

Em conflito entre pai e filho que marcou uma geração de jovens urbanos brasileiros. 0 próprio título original do filme já remete para um perfil de jovem ainda está presente em nossas cidades: Um rebelde sem causa. Jim Stark é preso por embriaguez e desordem. Na delegacia, conhece Judy, uma jovem que foi chamada de vagabunda pelo pai, e John Crawford, que deu uns tiros em uns cães. Na delegcia tudo parece c aminhar para uma solução. Mas no dia seguinte as coisas mudam.

\section{O SELVAGEM}

DIREÇÃO: LÁSZLÓ BENEDEK

atores: Marlon Brando (Johnny Strabler / Narrador), Mary Murphy (Kathie Bleeker), Robert Keith (Xerife Harry Bleeker), Lee Marvin (Chino), Jay C. Flippen (Xerife Stew Singer), Peggr Maley (Mildred), Hugh Sanders (Charlie Thomas), Ray Teal (Tio Frank Bleeker), John Brown (Bill hamegan).

DRAMA, EUA, 1953, 80 MINUTOS, BRANCO E PRETO.

Baseado no livro “The Cyclists' Raid” de Frank Rooney, "O Selvagem" conta um fato ocorrido em 1947, na pequena cidade de Hollister, na Califórnia, quando ela foi invadida por um grupo de cerca de 4.000 motoqueiros. Evidentemente, aqui o número é bem menor. Mas há um conflito entre duas gangues de motociclistas, numa cidade do interior dos Estados Unidos. O líder do bando, Johnny se apaixona pela filha do xerife local e as confusões aumentam com a chegada de uma gangue rival. Gestos e roupas foram copiados por muitos jovens, em todo o mundo. Mostra o início de uma época na qual a motocicleta representava um dos símbolos da insatisfação e rebelião dos jovens.

\section{OS 12 TRABALHOS}

DIREÇÃO: RICARDO ELIAS

atores: Sidney Santiago (HÉracles), Flávio Bauraqui (Jonas), Vera Mancini (Roseli), Vanessa Giácomo (Simone), Francisca Queiroz (Francisca), Cynthia Falabella (Gêmeas), Cacá Amaral (Sr. Ernesto), lucinha lins (Carmem), luiz Baccelli (Seu Moreira), André luís Patrício (Maguila), Eduardo Mancini (Mano Véıo), Ígor Zuvela (Enfermeiro), luciano Carvalho (Doze Pino), Thiago Moraes (Marcinho), Paulo américo (Catatau)

DRAMA, BRASIL, 2007, 90 MINUTOS, CORES.

Os percalços de um inexperiente ex-interno da Febem, ao conseguir um serviço de motoboy. Emergem preconceitos e dificuldades, quando ele tem que realizar 12 trabalhos para se efetivar na função. Um bom retrato da cidade opressora e da dificuldade que muitos adolescentes têm de lidar com ela.

\section{DOIS CURTAS-METRAGENS}

\section{RAÇA, RITMO E POESIA}

DIREÇÃO: MIRO NALLES

DOCUMENTÁRIO, BRASIL, 1994, 17 MINUTOS, CORES.

A partir de depoimentos de rappers de diferentes bairros da cidade de São Paulo, são conhecidos os trabalhos desenvolvidos por esses jovens na periferia, tendo como eixos de atuação a consciência, o ritmo e a poesia. 


\section{VINTE E DEZ: O FUTURO É AGORA.}

DIREÇÃO: TATA AMARAL E FRANCISCO CÉSAR FILHO

DOCUMENTÁRIO, BRASIL, 2002, 26. MINUTOS, CORES.

Deividson é um jovem de periferia de Santo André, região metropolitana de São Paulo, que manda seu recado para a comunidade através do Rap.

\section{LIVROS}

\section{ADOLESCENTES / MARTA REZENDE CARDOSO (ORG.)}

Editora Escuta, São Paulo, SP, 216 PÁGinas, 2006.

A adolescência é um processo marcante no desenvolvimento da subjetividade. Experiência de ruptura e transformação, a passagem da adolescência constitui um enigma para os que se dedicam a compreender o ser humano e as múltiplas humanidades. Este livro conta com a colaboração de Helena Aguiar, Mariana Barbosa, Joel. Um grupo de alunos contribuiu para a realização da pesquisa.

O psicanalista Pedro Henrique Bernardes Rondon comenta o livro da doutora Marta Rezende Cardoso:

Esta é a terceira coletânea de trabalhos sobre a adolescência que a professora doutora Marta Rezende Cardoso organiza, dentro do projeto de pesquisa que coordena sobre o tema no Instituto de Psicologia da UFRJ. Além das duas coletâneas anteriores (Adolescência: reflexões psicanalíticas. Rio de Janeiro: Nau/Faperj, 1999, e Limites. São Paulo: Escuta, 2004), esta pesquisa também já rendeu um número especial sobre violência psíquica da Pulsional Revista de Psicanálise (São Paulo, ano XV, n.163, novembro de 2002).

Em Adolescentes, estão reunidos artigos de diversos pesquisadores que têm se dedicado às questões dessa importante fase do desenvolvimento psíquico, especialmente do ponto de vista das chamadas patologias contemporâneas.

\section{JOVENS NA METRÓPOLE - ETNOGRAFIAS DE CIRCUITOS DE LAZER, ENCONTRO E SOCIABILIDADE / JOSÉ GUILHERME C. MAGNANI E BRUNA MANTESE DE SOUZA (ORG.)}

Editora Terceiro nome, São Paulo, SP, 280 páginas, 2007.

Straight edges, góticos, pichadores, japas e manos, baladeiros e instrumentistas, baladeiros do Senhor, baladeiros black e das rodas de samba, forrozeiros. Além de um relato saboroso apresentando várias tribos e/ou grupos urbanos, Jovens na Metrópole apresenta conceitos capazes de nos dar uma excelente compreensão do cenário da cidade grande. O livro é resultado de uma pesquisa realizada pelo Núcleo de Antropologia Urbana da Universidade de São Paulo - NAU.

\footnotetext{
JUVENTUDE - ENSAIOS SOBRE SOCIOLOGIA E HISTÓRIA DAS JUVENTUDES MODERNAS /

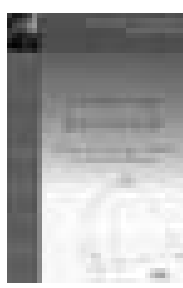
LUÍS ANTONIO GROPPO

Editora Difel, Rio de Janeiro, RJ, 308 PÁGinas, 2000.

Este cuidadoso trabalho de Luís Antônio Groppo nos apresenta um resumo da formação da juventude moderna. Ele reflete:

... a emergência da juventude como signo e a substituição da experiência juvenil pela vivência da “juvenilidade” podem ser explicadas pela própria atuação das juventudes e seus movimentos na Revolução Cultural da segunda metade do século XX

- cujo momento mais visível foram os anos 1960. Nessa Revolução Cultural, uma contradição recorrente da juventude moderna se explicitou e, talvez, solucionou-se: o papel transitório da juventude versus as identidades e as subculturas juvenis.
} 
Groppo trabalha com três noções para conceituar juventude. A noção de transitoriedade, na qual antecede a vida social plena; a noção de projeto, como estágio de preparação para uma vida posterior plena; e a noção de crise e ruptura. São analisados os rebeldes sem causa, a juventude nazista e fascista, as gangues urbanas e as revolucionárias.

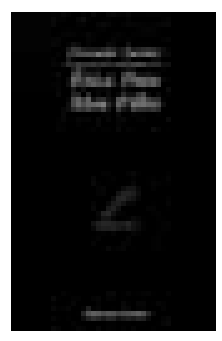

\section{ÉTICA PARA MEU FILHO / FERNANDO SAVATER}

TRADUÇÃo dE MÔNICA StAHEL Editora Martins Fontes, SÃo Paulo, SP, 180 PÁginas, SEgunda EdiçÃo, 1996.

Catedrático de Ética na Universidade do País Basco, Espanha, o filósofo Fernando Savater construiu uma maneira rigorosa, série e palatável de tratar de sua especialidade com o objetivo de falar para jovens, principalmente, e também para seus pais e professores. Ele mesmo adverte:

Este livro não é um manual de ética para alunos do colegial. Não contém informações sobre os autores mais notáveis e movimentos mais importantes da teoria moral ao longo da história. [...] Também não se trata de um receituário de respostas moralizantes aos problemas cotidianos que podemos encontrar no jornal e na rua, do aborto à objeção de consciência, passando pelo preservativo. [...] Seu objetivo não é fabricar cidadãos bem-pensantes (muito menos malapensados), mas estimular o desenvolvimento de livres-pensadores. Realmente, este livro é um bom estímulo para isso.

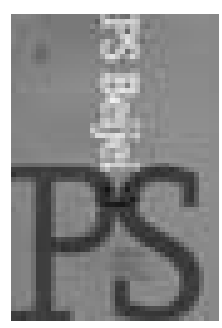

\section{PS BEIJEI / ADRIANA FALCÃO E MARIANA VERÍSSIMO}

Editora Salamandra, São Paulo, SP, 128 PÁGinas, 2004.

Eu nunca podia imaginar que o meu primeiro beijo ia ser numa segunda-feira.

Pouca gente no Brasil escreve tão bem, e tão criativamente, quanto Adriana Falcão. E, a julgar pela convivência, Mariana Veríssimo está em um ótimo caminho, muito bem acompanhada.

E a prova começa com este livro, uma divertida e inteligente correspondência entre duas adolescentes, que quebram o tédio de umas férias insólitas trocando confidências nas quais os destaques são... meninos. Um livro para todo mundo, principalmente para quem quer conhecer a formação do jeito de pensar feminino.

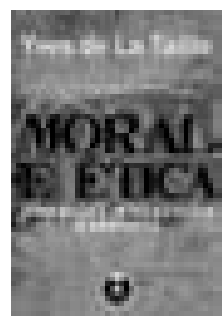

\section{MORAL E ÉTICA - DIMENSÕES INTELECTUAIS E AFETIVAS / YVES DE LA TAILLE}

Editora Artmed, São Paulo, SP, 192 PÁginas, 2006.

Yves de La Taille, professor de psicologia da USP, é bastante conhecido. Esta obra proporciona um conhecimento que fundamenta historicamente os conceitos de moral e ética e, ao mesmo tempo, complementa e enriquece o livro de Fernando Savater, também indicado.

Para este Mosaico, contudo, uma parte do livro de La Taille chama a atenção: no seu apêndice, ele apresenta a pesquisa: "Valores dos jovens de São Paulo".

0 autor fala de seu trabalho:

[...] o aumento da violência e da incivilidade, o consumo crescente de drogas, a grande freqüência de suicídios (que matam, no mundo, tantas pessoas quanto as guerras e os crimes somados), a tendência do "fechamento comunitário" nas grandes cidades, ao consumismo, à busca de divertimento [...], o desafeto pelo saber e a atividade intelectual \{...\} parecem ser indícios de um mal-estar ético.

Segundo La Taille, o objetivo desta pesquisa é procurar entender as causas desse mal-estar ético, sobretudo para subsidiar políticas públicas para a educação de c rianças e jovens. 


\section{SITES}

\section{Duas edições especiais sobre juventude}

\section{http://www.scielo.br/scielo.php?script=sci_issuetoc\&pid=0103-207020050002\&lng=pt\&nrm=iso}

REVISTA TEMPO SOCIAL V.17 N.2 SÃO PAULO NOV. 2005

JUVENTUDE(S) E TRANSIÇÕES

Heloísa Helena T. de Souza Martins e Maria Helena Augusto apresentam a publicação:

A organização, neste número da revista Tempo Social, de um dossiê sobre juventude tem o objetivo de retomar um tema que esteve presente nas preocupações de dois dos mais importantes sociólogos de nosso Departamento de Sociologia. O pequeno mas significativo estudo de Octavio lanni, O jovem radical, e as pesquisas de Marialice Foracchi sobre o estudante universitário e o movimento estudantil evidenciam a preocupação de compreender a sociedade brasileira tomando a categoria juventude como referência para estudar as transformações decorrentes do processo de desenvolvimento, a crise social e o papel político do jovem.

Estes são alguns autores e seus textos:

Para um novo significado do futuro: mudança social, jovens e tempo - Carmen Leccardi; O sentido do risco - Salvatore La Mendola; A face oculta da transferência de renda para jovens no Brasil - Marilia Pontes Sposito e Maria Clara Corrochano; Os circuitos dos jovens urbanos - José Guilherme Cantor Magnani; Juventude brasileira, entre a tradição e a modernidade - Hebe Signorini Gonçalves; Entrevistas com Paul Willis feitas por Roger Martinez e Melissa Mattos Pimenta; Retratos da juventude brasileira: análises de uma pesquisa nacional - Cristiane A. Fernandes da Silva.

\section{http://www.proec.ufg.br/revista_ufg/juventude/sumario.html}

REVISTA DA UFG - NÚMERO ESPECIAL JUVENTUDE

ÓRGÃO DE DIVULGAÇÃO DA UNIVERSIDADE FEDERAL DE GOIÁS - ANO VI, NO. 1, JUNHO DE 2004

No editorial da publicação, Múltiplos olhares sobre a juventude, Margareth Cavalcante de Castro Lobato comenta:

O ideal juvenil repousa no imaginário coletivo revelando-se ora através do desejo de ser jovem para sempre, ora através da percepção da juventude como um estado de espírito permanente. À revelia da luta diária que travamos com nosso relógio biológico, estendemos este ideal para as faixas etárias vindouras, alimentando o mito da juventude como condição para a felicidade eterna. Atribuímos-lhe um poder auto-realizante, que de fato não se sustenta, não resiste às investigações calcadas na realidade.

Alguns artigos desta edição:

Por uma história da juventude brasileira - Fernanda Quixabeira Machado; Contribuições da literatura do devaneio para o conceito de juventude - Jorge Alves Santana; O que eles falam sobre o jovem não é sério: uma crítica à perspectiva adultocêntrica da juventude - Sandra Unbehaum, Sylvia Cavasin, Valéria Nanci Silva; Tentativa de suicídio na adolescência - Célia Maria Ferreira da Silva Teixeira; A juventude e suas representações - Loriza Lacerda de Almeida. 


\section{Antropologia da juventude urbana}

\section{http://www.n-a-u.org/}

NÚCLEO DE ANTROPOLOGIA URBANA DA USP

O NAU, Núcleo de Antropologia Urbana, formado em 1988 no Departamento de Antropologia da USP, é um grupo de pesquisa e discussões teórico-metodológicas sobre questões relativas às sociedades urbano-industriais contemporâneas.

O Núcleo integra pesquisadores nos níveis de iniciação científica, mestrado e doutorado que se distribuem em quatro linhas temáticas: Práticas culturais e sociabilidade no contexto urbano, Formas de religiosidade, Metodos em Antropologia Urbana e Antropologia das sociedades complexas.

Além de estudos localizados na cidade de São Paulo, há trabalhos que foram ou estão sendo desenvolvidos em outras cidades e centros urbanos brasileiros como Florianópolis, Belém, Curitiba, Natal, São Carlos (SP) e Londrina (PR).

Destaque para o artigo:

Muitas palavras: a discussão recente sobre juventude nas Ciências Sociais, de Alexandre Barbosa Pereira, no Ponto Urbe, Revista do Núcleo de Antropologia urbana da USP, Ano 1, Versão 0.1, 2007. 〈http://www.n-au.org/PEREIRA-a-2007.html>

\section{Sites que atuam com jovens}

\section{BOCADA FORTE}

WWW.BOCADAFORTE.COM.BR

Revista eletrônica, músicas, vídeos, dentre outros materiais sobre Hip Hop -

\section{CENPEC - CENTRO DE ESTUdOS E PESQUISAS EM EDUCAÇÃO, CULTURA E AÇÃO COMUNITÁRIA}

WWW.CENPEC.ORG.BR

Desde 1987, o Cenpec atua em programas e projetos que auxiliam as políticas públicas em educação. Por meio de parcerias com o poder público, em todos os seus níveis, e com instituições empresariais de forte relevância social, o Cenpec vem ajudando a melhorar o desempenho educacional do Brasil.

Os principais projetos do Cenpec estão ligados ao desenvolvimento da educação pública, por meio de apoio direto a ações no espaço escolar ou em ambiente social com a participação de organizações parceiras.

Os programas e projetos do Cenpec chegam a mais de 3 mil municípios brasileiros e beneficiaram nestes 18 anos estudantes e professores em quase todos os Estados brasileiros.

\section{CIDADE ESCOLA APRENDIZ}

WWW.APRENDIZ.ORG.BR

Há dez anos, na Vila Madalena, bairro da cidade de São Paulo, o Aprendiz trabalha para tecer relações, articular esforços, gerar iniciativas, envolver agentes e serviços - tudo em prol da garantia de uma atenção integral à criança, ao adolescente e ao jovem. 


\section{EDUCAREDE}

WWW.EDUCAREDE.ORG.BR

Um dos mais importantes portais de educação no Brasil, patrocinado pela Telefônica. Oferece conteúdo para aprimorar professores e alunos.

\section{MICROFONIA}

WWW.MICROFONIA.COM

Eventos e produções independentes da cena punk.

\section{MUSEU DA PESSOA}

WWW.MUSEUDAPESSOA.COM.BR

Este é um museu virtual de histórias de vida aberto à participação gratuita de toda pessoa que queira compartilhar sua história. O Museu foi fundado em 1991. Desde o início, o objetivo era construir uma rede de histórias de vida que contribuísse para a transformação social.

\section{OBSERVATÓRIO JOVEM DO RIO DE JANEIRO}

WWW.UFF.BR/OBSJOVEM

Realiza estudos, pesquisas e atividades de extensão relacionadas com as transformações da condição juvenil na história, as situações de vida dos jovens na contemporaneidade e suas mobilizações sociais, culturais e políticas e acompanha criticamente o desenvolvimento das políticas públicas dirigidas aos jovens. Tem duas linhas de pesquisa: a) Juventude e processos educativos e b) Juventude e culturas da participação.

\section{ORGANIZAÇÃO BRASILEIRA DA JUVENTUDE}

WWW.OBJ.ORG.BR

A principal missão da OBJ é contribuir com a efetiva participação da juventude no desenvolvimento de nossa sociedade. Para isso, fomenta a implantação de políticas de juventude e a promoção dos direitos dos jovens, objetivando o desenvolvimento econômico e social sustentável da juventude brasileira.

\section{PORTAL DO PROTAGONISMO JUVENIL}

WWW.PROTAGONISMOJUVENIL.ORG.BR

O Protagonismo Juvenil é um tipo de ação de intervenção no contexto social para responder a problemas reais onde o jovem é sempre o ator principal. O Protagonismo Juvenil significa, tecnicamente, o jovem participar como ator principal em ações que não dizem respeito à sua vida privada, familiar e afetiva, mas a problemas relativos ao bem comum, na escola, na comunidade ou na sociedade mais ampla. Outro aspecto do protagonismo é a concepção do jovem como fonte de iniciativa, que é ação; como fonte de liberdade, que é opção; e como fonte de compromissos, que é responsabilidade.

\section{REAL HIP HOP}

WWW.REALHIPHOP.COM.BR

Portal fruto da iniciativa de vários jovens, envolvidos e não envolvidos com o movimento Hip Hop.

\section{UNESCO - ORGANIZAÇÃO DAS NAÇÕES UNIDAS PARA A EDUCAÇÃO, A CIÊNCIA E A CULTURA}

www.unesco.org.br 



\section{Cadernos Cenpec}

ANO 3

NÚMERO 5

PRIMEIRO SEMESTRE DE 2008

Cadernos Cenpec é uma publicação do

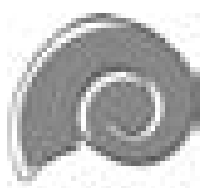

\section{CENPEC}

Cenpec - Centro de Estudos e Pesquisas em Educação, Cultura e Ação Comunitária

Rua Dante Carraro, 68

05422-060 - São Paulo - SP

Brasil

Telefax: (55) (11) 21329000

cenpec@cenpec.org.br

www.cenpec.org.br

Os artigos assinados não representam necessariamente os pontos de vista do CENPEC.

As opiniões e idéias expressas neles são de responsabilidade exclusiva de seus autores.

Cadernos Cenpec / Centro de Estudos e Pesquisas

em Educação, Cultura e Ação

Comunitária. - N. 5 (2008) -

São Paulo: CENPEC, 2006

ISSN 1808-9631

Semestral

1. Educação 5. CENPEC

\section{CenPeC}

Centro de Estudos e Pesquisas em Educação, Cultura e Ação Comunitária

\section{PRESIDÊNCIA}

Maria Alice Setubal Diretora Presidente

Ricardo Campus Caluby Ariani Diretor Vice-Presidente

Diretores Administrativos

LYDIA MARIA QUEIROZ FERREIRA DE MAGALHÃES

TEREZA MARIA MACEDO SOARES DE ARAÚJO

Conselho de Administração

Antonio Carlos Caruso Ronca

BERNADETE ANGELINA GATTI

HÉLIO MATTAR

Maria Alice Setubal

Michel PAUL ZEITLIN

Ricardo Campos CaIUby ARIANI

Conselho Fiscal

Reginaldo José CAMILO

Rebecca de Castro Filgueiras Raposo

\section{COORDENAÇÃo}

Coordenadora Geral

Maria do Carmo Brant de Carvalho

Assessoria da Coordenação

MARIA ÂNGELA LEAL RUdge

MARIA CRISTINA S. ZELMANOVITS

Maria AMABILE MansutTI

Coordenadora da Área Educação e Comunidade

MARIA JULIA AZEVEDo

Coordenadora Administrativo-Financeira

MARIA APARECIDA ACUNZO FORLI

Créditos desta edição

Organização e Coordenação

ISA MARIA F. ROSA GUARÁ

Comitê Editorial

ANA Regina CARRARA

ELOÍSA DE BLASIS

FERNANDO RIOS

ISA MARIA F. Rosa GUará

CDD 370

Maria do Carmo Brant de Carvalho 
Conselho Editorial

ÂMBAR DE BARROS

ANTONIO JACINTO MATHIAS

BERNADETE GATTI

FERnANDo ALmEIdA

FERNANDO ROSSETTI

Gilda Portugal Gouveia

ISA MARIA F. ROSA GUARÁ

Marco Aurélio Nogueira

MARia Alice Setubal

Maria do Carmo Brant de Carvalho

Vera Masagão Ribeiro

Equipe Jovens Urbanos

Coordenador Técnico - Wagner A. Santos

Núcleo Gerenciamento

HELENA F. CORRÊA

MARIA BRANT

ISABEL ARAÚJO - ESTAGIÁRIA

Núcleo Pesquisa

Aline Andrade

MARQUILANDES BORGES

MÔNICA MUSSI - CONSULTORA

Núcleo Formação

Eder CAMARgo

JuLIo NeVES

MARCo DALAMA

Núcleo Sistema de Informações

JORDI NOVAS

THIAGO CANTARIM

CLAudia CharouX

Núcleo Relações Institucionais

RITA CARMONA

HELENA HYPóLITO

RENATO SOUZA

Colaboram nesta edição

ALINE ANDRADE

FERNANDO RIOS

IRINEU FRANCISCO BARRETO JR.

JosÉ EDUARDO DE ANDRADE

Julia Alves Marinho Rodrigues

MARIA CRISTINA ZELMANOVITS
MARIA do CARMo Brant de Carvalho

Maria Helena Guimarães de CAStro

MARIA INÊS FINI

MARIA JÚLIA AZEVEDO

MÔNICA MUSSI

SIMONE APARECIDA JORGE

WAgner A. SANTOS

Redator

FERNANDO RIOS

Preparação e revisão de textos

ELIEL CUNHA

Projeto gráfico original

Homem de Melo \& Troia Design

Editoração eletrônica e ilustrações

FONTE DESIGN

Fotos

- CAPA, PÁGINAS 1, 6, 11, 13, 14, 15, 16, 19, 20, 21, 22, 25 , 26, 30, 31, 34, 37, 45, 46, 47, 48, 50, 51, 58, 65, 78, 82, 83, 96 E 112: FOTOMONTAGENS PRODUZIDAS NA OFICINA O JOVEM E O ESPAÇO URBANO (PJU - PROGRAMA JOVENS URBANOS/CENPEC) COORDENADA PELOS EDUCADORES DA ESTILO DE EDUCAR ASSESSORIA EDUCACIONAL - FRANCIELE BUSICO E WALDIR HERNANDES EM 2007. OS JOVENS PARTICIPANTES DA OFICINA SÃO: CARLOS SABINO DANTAS, Renata Veruska Barbosa Ferreira, Eva Maria V. de SÁ, DANILO dA S. AIOLFI, JáZIO Nascimento de Matos, Silvana Sales do Nascimento, Bruna Aparecida Barbosa Mendes, Daiane Demétrio de Paula, ANDERSON ROdRIGUES BERNARDO, VANESSA RODRIGUES da Silva, Thiago Macedo Vicente da Silva e Joice de Cássia Salles Silva

- PÁginas 52, 100 E 101: JOVEns Participantes da $2^{\text {a }}$ Edição (PJU-PRograma Jovens URBanos/CENPEC) PARA A ExposiçÃo Múltiplos Olhares da Brasilândia

- PÁGINAS 68, 69, 86, 87, 88, 89, 90, 91, 93: LUANA FISCHER

Tiragem

2.000 exemplares 


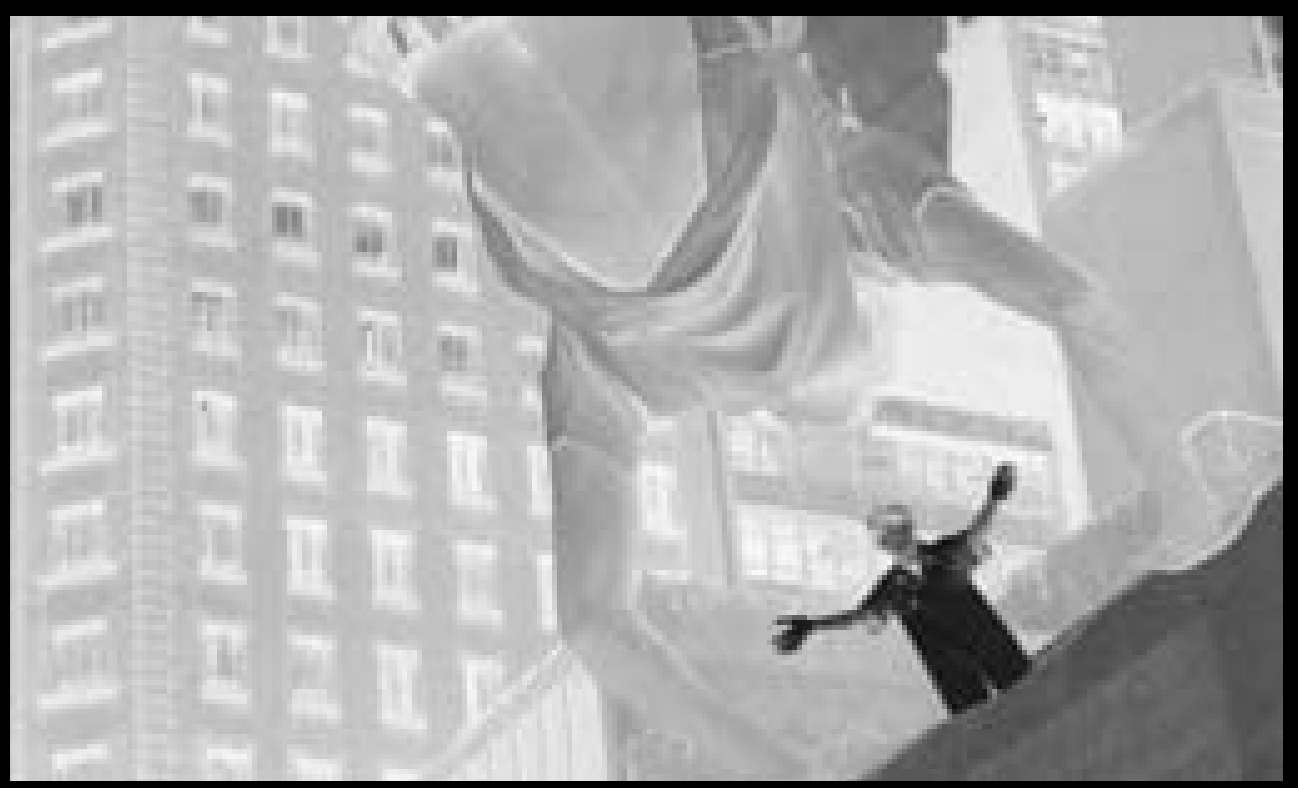

